\title{
Pullback attractors for non-autonomous
} reaction-diffusion equation in non-cylindrical domains

\section{Yanping Xiao* (1)}

\section{"Correspondence:} hardxiao@126.com College of Mathematics and Computer Science, Northwest University for Nationalities, Lanzhou, 730030, P.R. China

\begin{abstract}
In non-cylindrical domains, we use the definition of the variational solution and the maximum principle (the Galerkin method used usually in a cylindrical domain cannot be applied directly) to prove the existence of a pullback $\mathscr{D}_{\lambda_{1}}$ attractor in $L^{P}\left(\mathcal{O}_{t}\right)$ (any $p \geq 2$ ) for a reaction-diffusion equation. Then, with an appropriate assumption, the higher-order integrability of variational solution is obtained. Finally, we discuss the existence of a pullback $\mathscr{D}_{\lambda_{1}}$ attractor in $L^{2+\delta}\left(\mathcal{O}_{t}\right)$ for any $\delta \in[0,+\infty)$.
\end{abstract}

MSC: 35K57; 35B40; 35B41

Keywords: variational solution; pullback $\mathscr{D}_{\lambda_{1}}$ attractor; higher-order integrability; non-cylindrical domains

\section{Introduction}

The problems defined in cylindrical domains have been considered extensively. But for some phenomena, such as water waves representing rich phenomena on time-varying spatial domains, for example, the propagation of a tsunami across the open ocean (see [1]), the depth changes as the wave propagates toward the shore, which be seen as an example of a system with time-varying domain. In situations involving rapid temporal changes, such as those occurring during earthquakes or landslides, we may consider a time dependent depth. In other areas, there are a large number of biological processes involving non-cylindrical domains, such as the migrating range of species with season changing, mammalian coat patterns, skin patterns on tropical fish, solid tumor growth etc. Hence, it is necessary to study problems defined in domains varying with time.

Let $\left\{\mathcal{O}_{t}\right\}_{t \in \mathbb{R}}$ be a family of nonempty bounded open subsets of $\mathbb{R}^{N}$ such that

$$
\mathcal{O}_{s} \subset \mathcal{O}_{t}, \quad s<t
$$

Define

$$
Q_{\tau, T}:=\bigcup_{t \in(\tau, T)} \mathcal{O}_{t} \times\{t\}, \quad \tilde{Q}_{\tau, T}:=\bigcup_{t \in(\tau, T)} \mathcal{O}_{T} \times\{t\} \quad \text { for any } T>\tau,
$$

(c) 2016 Xiao. This article is distributed under the terms of the Creative Commons Attribution 4.0 International License (http://creativecommons.org/licenses/by/4.0/), which permits unrestricted use, distribution, and reproduction in any medium, provided you give appropriate credit to the original author(s) and the source, provide a link to the Creative Commons license, and indicate if changes were made. 
and

$$
\begin{aligned}
& Q_{\tau}:=\bigcup_{t \in(\tau,+\infty)} \mathcal{O}_{t} \times\{t\}, \quad \forall \tau \in \mathbb{R}, \\
& \Sigma_{\tau, T}:=\bigcup_{t \in(\tau, T)} \partial \mathcal{O}_{t} \times\{t\}, \quad \forall \tau<T \quad \text { and } \quad \Sigma_{\tau}:=\bigcup_{t \in(\tau,+\infty)} \partial \mathcal{O}_{t} \times\{t\} .
\end{aligned}
$$

We consider the following initial boundary value problem with homogeneous Dirichlet boundary condition:

$$
\left\{\begin{array}{l}
\frac{\partial u}{\partial t}-\Delta u+g(u)=f(t) \quad \text { in } Q_{\tau}, \\
u=0 \text { on } \Sigma_{\tau}, \\
u(\tau, x)=u_{\tau}(x), \quad x \in \mathcal{O}_{\tau},
\end{array}\right.
$$

where $u_{\tau}: \mathcal{O}_{\tau} \rightarrow \mathbb{R}$ and $f: Q_{\tau} \rightarrow \mathbb{R}$ are given for $\tau \in \mathbb{R}$, and $g \in C^{1}(\mathbb{R}, \mathbb{R})$ satisfies the conditions that there exist nonnegative constants $\alpha_{1}, \alpha_{2}, \beta, l$, and $p \geq 2$, such that

$$
-\beta+\alpha_{1}|s|^{p} \leq g(s) s \leq \beta+\alpha_{2}|s|^{p}, \quad \forall s \in \mathbb{R}
$$

and

$$
g^{\prime}(s) \geq-l, \quad \forall s \in \mathbb{R}
$$

For each $T>\tau$, consider the auxiliary problem

$$
\left\{\begin{array}{l}
\frac{\partial u}{\partial t}-\Delta u+g(u)=f(t) \quad \text { in } Q_{\tau, T} \\
u=0 \text { on } \Sigma_{\tau, T}, \\
u(\tau, x)=u_{\tau}(x), \quad x \in \mathcal{O}_{\tau}
\end{array}\right.
$$

where $u_{\tau}: \mathcal{O}_{\tau} \rightarrow \mathbb{R}$ for $\tau \in \mathbb{R}, g$ satisfies (1.4)-(1.5), and $f: Q_{\tau, T} \rightarrow \mathbb{R}$.

When the domains vary with respect to time, even though the forcing term $f$ is independent of time, the problem is still non-autonomous.

For domains $\mathcal{O}_{t} \equiv \mathcal{O}, t \in \mathbb{R}$, (1.3) returns to the usual non-autonomous reactiondiffusion equation which was studied extensively (see, for example, [2-7]). Specially, we have [5] obtained a pullback attractor in $L^{p}(\mathcal{O})(p$ comes from $(1.4))$ by requiring

$$
\|f\|_{L^{2}(\mathcal{O})} \leq C_{1} e^{\alpha|t|}, \quad \forall t \in \mathbb{R},
$$

where $0 \leq \alpha<\lambda, \lambda$ is the first eigenvalue of $-\Delta$ in $H_{0}^{1}(\mathcal{O})$. Recently, Łukaszewicz in [4] applying the Galerkin method and the Gronwall lemma introduced in [3] with forcing terms $f \in L_{\text {loc }}^{2}\left(\mathbb{R} ; L^{2}(\mathcal{O})\right)$ and

$$
\int_{-\infty}^{t} e^{\lambda s}\|f(s)\|_{L^{2}(\mathcal{O})}^{2} d s<\infty
$$

considered the existence of pullback $\mathscr{D}$ attractor in $L^{p}$, which, to the best of our knowledge, is the best result. 
For the case of domains changing in time, one may refer to [8-14] and the references therein. Specially, for domains expanding in time (that is, the condition (1.1)), by using a penalty method, the authors in [10] considered the existence and uniqueness of a variational solution for $(1.3)$ and obtained $\left(L^{2}\left(\mathcal{O}_{\tau}\right), L^{2}\left(\mathcal{O}_{t}\right)\right)$ pullback $\mathscr{D}_{\lambda_{1}}$ attractor. By applying a diffeomorphism, [11] obtained the existence and uniqueness of a weak solution for (1.3), furthermore, one proved the existence of a $\left(L^{2}\left(\mathcal{O}_{\tau}\right), L^{2}\left(\mathcal{O}_{t}\right)\right)$ pullback $\mathscr{D}$ attractor.

Then, as the problem defined in a cylindrical domain, we may ask whether the pullback attractor in $L^{p}\left(\mathcal{O}_{t}\right)$ exists as well; moreover, for any $\delta \geq 0$, what is the result as regards the pullback attractor in $L^{2+\delta}\left(\mathcal{O}_{t}\right)$ ?

In cylindrical domains, by applying the Garlerkin method and selecting $\left|(u-M)_{+}\right|^{p-2}(u-$ $M)_{+}$as a testing function, the pullback attractor in $L^{p}$ has been proved (see [4-6]). But in non-cylindrical domains, due to the characteristic of the penalty term (which is defined in Hilbert space), it is difficult or even impossible to choose $\left|(u-M)_{+}\right|^{p-2}(u-M)_{+}$ as a testing function, so the method in [4] cannot be applied directly to deduce the existence of pullback attractor in $L^{p}\left(\mathcal{O}_{t}\right)$. On the other hand, when we have a forcing term $f \in L_{\text {loc }}^{2}\left(\mathbb{R} ; L^{2}\left(\mathcal{O}_{t}\right)\right)$, it is impossible to obtain a solution $u \in L^{2+\delta}\left(\mathcal{O}_{t}\right)$ for any $\delta \leq 0$. Based on the existence of the $L^{2}\left(\mathcal{O}_{t}\right)$ pullback $\mathscr{D}_{\lambda_{1}}$ attractor, the authors in [13] provided a new method to show that the pullback $\mathscr{D}_{\lambda_{1}}$ attractor in $L^{2}\left(\mathcal{O}_{t}\right)$ can attract sets in the topology of $L^{2+\delta}\left(\mathcal{O}_{t}\right)(\forall \delta \in[0, \infty))$. But the existence of a pullback $\mathscr{D}_{\lambda_{1}}$ attractor in $L^{2+\delta}\left(\mathcal{O}_{t}\right)$ is still unknown.

In this paper, by applying the definition of a variational solution and a maximum principle, we first consider the existence of a pullback $\mathscr{D}_{\lambda_{1}}$ attractor in $L^{p}\left(\mathcal{O}_{t}\right)$; then, as a corollary, with the forcing term satisfying $f \in L_{\text {loc }}^{\infty}\left(\mathbb{R}^{N+1}\right)$, we establish the existence of the pullback $\mathscr{D}_{\lambda_{1}}$ attractor in $L^{2+\delta}\left(\mathcal{O}_{t}\right)(\delta \in[0,+\infty))$ for a non-autonomous reaction-diffusion equation defined in non-cylindrical domains. The main results are the following.

Theorem 1.1 Suppose (1.1), (1.2), (1.4), and (1.5) hold, $u_{\tau} \in L^{2}\left(\mathcal{O}_{\tau}\right)$ and $f \in L_{\mathrm{loc}}^{2}\left(\mathbb{R}^{N+1}\right)$ satisfying

$$
\int_{-\infty}^{t} e^{\lambda_{1}, t}|f(r)|_{r}^{2} d r<+\infty \quad \text { for all } t \in \mathbb{R}
$$

here $\lambda_{1, t}$ is the first eigenvalue of $-\Delta$ in $H_{0}^{1}\left(\mathcal{O}_{t}\right)$. Then the process $U(\cdot, \cdot)$ corresponding to problem (1.3) has a minimal unique pullback $\mathscr{D}_{\lambda_{1}}$ attractors $\mathscr{A}(t)$ in $L^{p}\left(\mathcal{O}_{t}\right)$ (p comes from (1.4)) for all $t \in \mathbb{R}$.

Theorem 1.2 Suppose that (1.1), (1.2), (1.4), and (1.5) hold, $u_{\tau} \in L^{2}\left(\mathcal{O}_{\tau}\right)$ and $f \in L_{\mathrm{loc}}^{\infty}\left(\mathbb{R}^{N+1}\right)$. Let $U(\cdot, \cdot)$ be the process generated by the variational solutions of (1.3). Then, for any $\delta \in$ $[0, \infty)$, there exists a pullback $\mathscr{D}_{\lambda_{1}}$ attractor $\hat{\mathscr{A}}_{\text {in }} L^{2+\delta}\left(\mathcal{O}_{t}\right)$, that is, $\hat{\mathscr{A}}$ is compact, invariant and we have a pullback $\mathscr{D}_{\lambda_{1}}$ attracting in the topology of $L^{2+\delta}\left(\mathcal{O}_{t}\right)$ for any $\delta \in[0,+\infty)$.

This paper is organized as follows. In Section 2, we will present some definitions, and related results as regards the pullback attractor and a variational solution. By means of the definition of the variational solution and the maximum principle, in Section 3, the pullback $\mathscr{D}_{\lambda_{1}}$ attractor in $L^{p}\left(\mathcal{O}_{t}\right)$ is established (see Theorem 1.1). In Section 4, the higherorder integrability of the approximation solution and the variational solution associated to (1.3) are obtained. Furthermore, we discuss the existence of a pullback $\mathscr{D}_{\lambda_{1}}$ attractor in $L^{2+\delta}\left(\mathcal{O}_{t}\right)(\delta \in[0,+\infty))$ (see Theorem 1.2). 


\section{Preliminaries}

For each $t \in \mathbb{R}$, we denote by $(\cdot, \cdot)_{t}$ and $|\cdot|_{t}$ the usual inner product and related norm in $L^{2}\left(\mathcal{O}_{t}\right)$ and by $((\cdot, \cdot))_{t}$ and $\|\cdot\|_{t}$ the usual gradient inner product and associated norm in $H_{0}^{1}\left(\mathcal{O}_{t}\right)$. The usual duality product between $H_{0}^{1}\left(\mathcal{O}_{t}\right)$ and $H^{-1}\left(\mathcal{O}_{t}\right)$ will be denoted by $\langle\cdot, \cdot\rangle_{t}$. Also $(\cdot, \cdot)_{t}$ and $\|\cdot\|_{L^{p}\left(\mathcal{O}_{t}\right)}$ represent the duality product between $L^{p}\left(\mathcal{O}_{t}\right)$ and $L^{q}\left(\mathcal{O}_{t}\right)$ with $\frac{1}{p}+\frac{1}{q}=1$ and associated norm.

\subsection{Pullback attractor}

We will recall some concepts about pullback attractor which can be found, for example, in $[6,10]$.

We consider a process (also called a two-parameter semigroup) $U$ on a Banach space $X$, i.e. a family $\{U(t, \tau) ;-\infty<\tau \leq t<+\infty\}$ of continuous mappings $U(t, \tau): X \rightarrow X$, such that

$$
U(\tau, \tau) x=x \quad \text { and } \quad U(t, \tau)=U(t, s) U(s, \tau) \quad \text { for all } \tau \leq s \leq t \text { and } x \in X .
$$

Suppose $\mathscr{D}$ is a nonempty class of parameterized sets $\hat{D}=\{D(t): t \in \mathbb{R}\} \subset \mathcal{P}(X)$, where $\mathcal{P}(X)$ denotes the family of all nonempty subsets of $X$.

Definition 2.1 It is said that $\hat{B} \in \mathscr{D}$ is pullback $\mathscr{D}$-absorbing for the process $U(\cdot, \cdot)$ if for any $t \in \mathbb{R}$ and any $\hat{D} \in \mathscr{D}$, there exists a $\tau_{0}=\tau_{0}(t, \hat{D}) \leq t$ such that

$$
U(t, \tau) D(\tau) \subset B(t) \quad \text { for all } \tau \leq \tau_{0}(t, \hat{D}) .
$$

Definition 2.2 The process $U(\cdot, \cdot)$ is called norm-to-weak continuous if

$$
U(t, \tau) x_{n} \rightarrow U(t, \tau) x \quad \text { as } x_{n} \rightarrow x \text { in } X
$$

for all $t \geq \tau, \tau \in \mathbb{R}$, where $X$ is a Banach space.

Let $X$ be a complete metric space and $B$ be a bounded subset of $X$. The Kuratowskii measure of noncompactness $\alpha(B)$ of $B$ is defined by

$$
\alpha(B)=\inf \{\delta>0 \mid B \text { has a finite open cover of sets of diameter } \leq \delta\} .
$$

Definition 2.3 A process $\{U(t, \tau)\}$ is called pullback $\omega$ - $\mathscr{D}$-limit compact if for any $\epsilon>0$ and $\hat{D} \in \mathscr{D}$, there exists a $\tau_{0}(\hat{D}, t) \leq t$ such that $\alpha\left(\bigcup_{\tau \leq \tau_{0}} U(t, \tau) D(\tau)\right) \leq \epsilon$.

Theorem 2.4 Let $U(t, \tau)$ be a process in $X$ satisfying the following conditions:

(i) $U(t, \tau)$ is norm-to-weak continuous in $X$;

(ii) there exists a family $\hat{B}$ of pullback $\mathscr{D}$-absorbing sets in $X$;

(iii) $U(t, \tau)$ is pullback $\omega$-D -limit compact in $X$.

Then there exists a minimal pullback $\mathscr{D}$-attractor $\hat{A}$ in X given by

$$
\hat{\mathscr{A}}=\omega(\hat{B}, t)=\bigcap_{s \leq t} \overline{\bigcup_{\tau \leq s} U(s, \tau) B(\tau)} .
$$


By following a similar process to Lemma 2.1 in [4], we can get the result defined in noncylindrical domains; it is very useful for checking condition (iii) in Theorem 2.4 when the phase space is a Lebesgue space $L^{p}\left(\mathcal{O}_{t}\right)$.

Theorem 2.5 Let $U(t, \tau)$ be a process in $L^{p}\left(\mathcal{O}_{t}\right)$ and $L^{q}\left(\mathcal{O}_{t}\right)$ respectively, $p>q>0$, satisfying the following conditions:

(i) $U(t, \tau)$ is pullback $\omega$ - $\mathscr{D}$-limit compact in $L^{q}\left(\mathcal{O}_{t}\right)$;

(ii) for every $\varepsilon>0$ and $\hat{B} \in \mathscr{D}$, there exist $M=M(\varepsilon, \hat{B})$ and $\tau_{1}=\tau_{1}(\varepsilon, \hat{B})$ such that

$$
\int_{\left|U(t, \tau) u_{\tau}\right| \geq M}\left|U(t, \tau) u_{\tau}\right|^{p} d x<\varepsilon
$$

for any $u_{\tau} \in B(\tau), \tau \leq \tau_{1}$ and any $t \in \mathbb{R}$.

Then $U(t, \tau)$ is pullback $\omega$-D्D-limit compact in $L^{p}\left(\mathcal{O}_{t}\right)$.

Theorem 2.6 ([15]) Let $X, Y$ be two Banach spaces, $X^{*}, Y^{*}$ be respectively their dual spaces. Assume that $X$ is dense in $Y$, the injection $i: X \rightarrow Y$ is continuous, its adjoint $i^{*}: Y^{*} \rightarrow X^{*}$ is dense, and $U$ is a norm-to-weak continuous process on $Y$. Then $U$ is a norm-to-weak continuous process on $X$ if and only if, for any $\tau \in \mathbb{R}, t \geq \tau, U(t, \tau)$ maps compact sets of $X$ to bounded sets of $X$.

\subsection{Variational solutions for equation (1.6)}

As it is convenient for the application later, we recall some notations and variational solutions of (1.6) given by [10].

For each $T>\tau$, denote

$$
\begin{aligned}
\mathcal{U}_{\tau, T}:= & \left\{\phi \in L^{2}\left(\tau, T ; H_{0}^{1}\left(\mathcal{O}_{T}\right)\right) \cap L^{p}\left(\tau, T ; L^{p}\left(\mathcal{O}_{T}\right)\right), \phi^{\prime} \in L^{2}\left(\tau, T ; L^{2}\left(\mathcal{O}_{T}\right)\right)\right. \\
& \text { and } \left.\phi(\tau)=\phi(T)=0, \phi(t) \in H_{0}^{1}\left(\mathcal{O}_{t}\right) \text { for a.e. } t \in(\tau, T)\right\} .
\end{aligned}
$$

Definition 2.7 A variational solution of equation (1.6) is a function $u$ such that

(C1) $u \in L^{2}\left(\tau, T ; H_{0}^{1}\left(\mathcal{O}_{T}\right)\right) \cap L^{p}\left(\tau, T ; L^{p}\left(\mathcal{O}_{T}\right)\right)$;

(C2) $u(t) \in H_{0}^{1}\left(\mathcal{O}_{t}\right)$ a.e. $t \in(\tau, T)$;

(C3) $\int_{\tau}^{T}\left[-\left(u(t), \phi^{\prime}(t)\right)_{T}+((u(t), \phi(t)))_{T}+(g(u(t)), \phi(t))_{T}\right] d t$

$$
=\int_{\tau}^{T}(f(t), \phi(t))_{T} d t \quad \text { for any } \phi \in \mathcal{U}_{\tau, T}
$$

(C4) $\lim _{t \rightarrow \tau} \frac{1}{t-\tau} \int_{\tau}^{t}|u(s)-u(\tau)|_{T}^{2} d s=0$.

Theorem 2.8 ([10]) Suppose that (1.1), (1.2), (1.4), and (1.5) hold, for $f \in L^{2}\left(\tau, T ; L^{2}\left(\mathcal{O}_{T}\right)\right)$ and $u_{\tau} \in L^{2}\left(\mathcal{O}_{\tau}\right)$, there exists a unique variational solution $u \in L^{2}\left(\tau, T ; H_{0}^{1}\left(\mathcal{O}_{T}\right)\right) \cap$ $L^{p}\left(\tau, T ; L^{p}\left(\mathcal{O}_{T}\right)\right)$ of equation (1.6) and the variational solution satisfies the energy equality a.e. $t \in[\tau, T]$, that is,

$$
|u(t)|_{T}^{2}+2 \int_{\tau}^{t}\|u(s)\|_{T}^{2} d s+2 \int_{\tau}^{t}(g(u(s)), u(s))_{T} d s=\left|u_{\tau}\right|_{T}^{2}+2 \int_{\tau}^{t}(f(s), u(s))_{T} d s
$$


holds for a.e. $t \in[\tau, T]$. In addition, $u \in C\left([\tau, T] ; L^{2}\left(\mathcal{O}_{T}\right)\right)$ and satisfies the energy equality for all $t \in[\tau, T]$. Moreover, if $u_{\tau} \in H_{0}^{1}\left(\mathcal{O}_{\tau}\right) \cap L^{p}\left(\mathcal{O}_{\tau}\right)$, then $u$ also satisfies

$$
u \in L^{\infty}\left(\tau, T ; H_{0}^{1}\left(\mathcal{O}_{T}\right)\right) \cap L^{\infty}\left(\tau, T ; L^{p}\left(\mathcal{O}_{T}\right)\right), \quad u^{\prime} \in L^{2}\left(\tau, T ; L^{2}\left(\mathcal{O}_{T}\right)\right) .
$$

Definition 2.9 ([10]) $u: Q_{\tau} \rightarrow \mathbb{R}$ is called a variational solution of (1.3), if, for any $T>\tau$, $u$ restricted in $Q_{\tau, T}$ is the variational solution of (1.6).

Let

$$
f \in L_{\mathrm{loc}}^{2}\left(\mathbb{R}^{N+1}\right) \text { and } \int_{-\infty}^{t} e^{\lambda_{1, t} s}|f(s)|_{s}^{2} d s<+\infty \quad \text { for any } t \in \mathbb{R} .
$$

Suppose $f$ satisfies (2.2), from Theorem 2.8 and Definition 2.9, for any $\tau \in \mathbb{R}$, any $u_{\tau} \in$ $\mathrm{七}^{2}\left(\mathcal{O}_{\tau}\right)$ and a.e. $t \in(\tau, T)(T>\tau)$, there exists a unique variational solution $u\left(\cdot ; \tau, u_{\tau}\right)$.

Let

$$
U(t, \tau) u_{\tau}:=u\left(t ; \tau, u_{\tau}\right), \quad-\infty<\tau \leq t<\infty, u_{\tau} \in L^{2}\left(\mathcal{O}_{\tau}\right)
$$

then (2.3) defines a process $U(\cdot, \cdot)$ of a family of set $\left\{u_{\tau} \in L^{2}\left(\mathcal{O}_{\tau}\right), \tau \in \mathbb{R}\right\}$.

Let $\mathcal{R}_{\lambda_{1}}$ be the set of functions $r: \mathbb{R} \rightarrow[0, \infty)$ satisfying

$$
\lim _{\tau \rightarrow-\infty} e^{\lambda_{1, t} \tau} r^{2}(\tau)=0
$$

where $\lambda_{1, t}$ is the first eigenvalue of $-\Delta$ in $H_{0}^{1}\left(\mathcal{O}_{t}\right)$. Denote by $\mathscr{D}_{\lambda_{1}}$ the family of $\hat{D}:=\{D(t) \subset$ $\left.L^{2}\left(\mathcal{O}_{t}\right), t \in \mathbb{R}\right\}$ such that

$$
D(t) \subset \bar{B}\left(0, r_{\hat{D}}(t)\right) \text { for some } r_{\hat{D}}(t) \in \mathcal{R}_{\lambda_{1}} \text {, }
$$

here $\bar{B}\left(0, r_{\hat{D}}(t)\right)$ is the closed ball with center in 0 and radius $r_{\hat{D}}(t)$.

Then, the following result holds.

Lemma 2.10 ([14]) Suppose (1.1), (1.2), (1.4), and (1.5) hold, $u_{\tau} \in L^{2}\left(\mathcal{O}_{\tau}\right)$ and $f$ satisfies (2.2). Then, process $U(\cdot, \cdot)$ associated to equation (1.3) generates a unique $\left(L^{2}\left(\mathcal{O}_{\tau}\right), L^{2}\left(\mathcal{O}_{t}\right)\right)$ pullback $\mathscr{D}_{\lambda_{1}}$ attractor $\hat{\mathscr{A}}$.

Together with Theorem 2.5 and Theorem 2.6 , we easily obtain the pullback $\mathscr{D}_{\lambda_{1}}$ attractor in $L^{p}(p>2)$ defined in domains increasing with time.

Theorem 2.11 Let $U(t, \tau)$ be a process in $L^{p}\left(\mathcal{O}_{t}\right)$ satisfying the following conditions:

(i) $U(t, \tau)$ is norm-to-weak continuous in $L^{p}\left(\mathcal{O}_{t}\right)$;

(ii) there exists a family $\hat{B}$ of pullback $\mathscr{D}_{\lambda_{1}}$-absorbing sets in $L^{p}\left(\mathcal{O}_{t}\right)$;

(iii) $U(t, \tau)$ is pullback $\omega$ - $\mathscr{D}_{\lambda_{1}}$-limit compact in $L^{p}\left(\mathcal{O}_{t}\right)$.

Then there exists a minimal pullback $\mathscr{D}_{\lambda_{1}}$-attractor $\hat{\mathscr{A}}$ in $L^{p}\left(\mathcal{O}_{t}\right)$ given by

$$
\hat{\mathscr{A}}=\omega(\hat{B}, t)=\bigcap_{s \leq t} \overline{\bigcup_{\tau \leq s} U(s, \tau) B(\tau)} .
$$


To make the test function used later meaningful, in [14], the following maximum principle holds.

Lemma 2.12 ([14] $)$ Suppose $u_{\tau} \in L^{\infty}\left(\mathcal{O}_{\tau}\right) \cap H_{0}^{1}\left(\mathcal{O}_{\tau}\right), f \in L^{\infty}\left(\tilde{Q}_{\tau, T}\right)$, and $g$ satisfies (1.4). Then there exists a positive constant $K$ depending on $\left\|u_{\tau}\right\|_{L^{\infty}\left(\mathcal{O}_{\tau}\right)},\|f\|_{L^{\infty}\left(\tilde{Q}_{\tau, T}\right)}, \beta$, and $\alpha_{1}$ such that the variational solution $u$ of equation (1.6) satisfies

$$
\|u\|_{L^{\infty}\left(\tilde{Q}_{\tau, T}\right)} \leq K
$$

\section{Pullback $\mathscr{D}_{\lambda_{1}}$ attractor in $L^{p}$}

In this section, by applying the definition of the variational solution of equation (1.6), we consider the existence of a pullback $\mathscr{D}_{\lambda_{1}}$ attractor in $L^{p}$ ( $p$ satisfying (1.4)).

Denote

$$
u(t)_{+}:=\left\{\begin{array}{ll}
u(t), & u(t) \geq 0, \\
0, & u(t)<0,
\end{array} \quad u(t)_{-}:= \begin{cases}u(t), & u(t) \leq 0 \\
0, & u(t)>0\end{cases}\right.
$$

Lemma 3.1 Assume $u_{\tau m} \in L^{\infty}\left(\mathcal{O}_{\tau}\right) \cap H_{0}^{1}\left(\mathcal{O}_{\tau}\right), f_{m} \in L_{\text {loc }}^{\infty}\left(\mathbb{R}, L^{\infty}\left(\mathcal{O}_{t}\right)\right)$, then the variational solution $u_{m}(t)$ of (1.6) satisfies the inequality

$$
\begin{aligned}
& \frac{d}{d t}\left\|\left(\left|u_{m}(t)\right|-M\right)_{+}\right\|_{L^{p}\left(\mathcal{O}_{T}\right)}^{p}+\frac{\alpha_{1} p}{4} M^{p-2} \int_{\mathcal{O}_{T}}\left|\left(\left|u_{m}(t)\right|-M\right)_{+}\right|^{p} d x \\
& \quad \leq C_{p, \alpha_{1}} \int_{\mathcal{O}_{T}}\left|f_{m}(t)\right|^{2} d x
\end{aligned}
$$

for a.e. $t \in(\tau, T)$.

Proof For any fixed $\tau \in(-\infty, T]$, denoted by $u_{m}(t)$ we have the variational solution of equation (1.6) corresponding to data $\left(u_{\tau m}, f_{m}\right)$ with

$$
u_{\tau m} \in L^{\infty}\left(\mathcal{O}_{\tau}\right) \cap H_{0}^{1}\left(\mathcal{O}_{\tau}\right) \text { and } f_{m} \in L^{\infty}\left(\tilde{Q}_{\tau, T}\right) .
$$

By Lemma 2.12 and Theorem 2.8, we know

$$
u_{m} \in L^{2}\left(\tau, T ; H_{0}^{1}\left(\mathcal{O}_{T}\right)\right) \cap L^{\infty}\left(\tilde{Q}_{\tau, T}\right)
$$

and for any $\eta \in C_{c}^{1}(\tau, T), \eta u_{m} \in \mathcal{U}_{\tau, T}$. According to the details of Lemma 2.12 (introduced in [14]), that is, denoting

$$
K^{\prime}:=\max \left\{\left\|u_{\tau m}\right\|_{L^{\infty}\left(\mathcal{O}_{\tau}\right)},\left\|f_{m}\right\|_{L^{\infty}\left(\tilde{Q}_{\tau, T}\right)}\right\}
$$

and by (1.4), we know there exists a positive constant $K^{\prime}$ depending on nonnegative constants $\beta, \alpha_{1}$, and a positive constant $M$, such that

$$
g(s)>K^{\prime}, \quad s \geq M \quad \text { or } \quad g(s)<-K^{\prime}, \quad s \leq-M .
$$

Defining

$$
K:=\max \left\{K^{\prime}, M\right\}+1
$$


we have $\left\|u_{m}\right\|_{L^{\infty}\left(\tilde{Q}_{\tau, T}\right)} \leq K$. Hence, we obtain

$$
\left|\left(u_{m}-M\right)_{+}\right|^{p-2}\left(u_{m}-M\right)_{+} \in L^{2}\left(\tau, T ; H_{0}^{1}\left(\mathcal{O}_{T}\right)\right) \cap L^{\infty}\left(\tilde{Q}_{\tau, T}\right)
$$

for the $M$ above and $p>2$, and for any $\eta \in C_{c}^{1}(\tau, T)$,

$$
\eta\left|\left(u_{m}-M\right)_{+}\right|^{p-2}\left(u_{m}-M\right)_{+} \in \mathcal{U}_{\tau, T} .
$$

So, according to the definition of a variational solution, we can choose

$$
\psi(t):=\eta\left|\left(u_{m}-M\right)_{+}\right|^{p-2}\left(u_{m}-M\right)_{+}
$$

as a testing function to obtain

$$
\int_{\tau}^{T}\left[-\left(u_{m}(t), \psi^{\prime}(t)\right)_{T}+\left(\left(u_{m}(t), \psi(t)\right)\right)_{T}+\left(g\left(u_{m}(t)\right), \psi(t)\right)_{T}\right] d t=\int_{\tau}^{T}\left(f_{m}(t), \psi(t)\right)_{T} d t
$$

therefore, for a.e. $t \in(\tau, T)$,

$$
\begin{aligned}
& \left(u_{m}^{\prime}(t),\left|\left(u_{m}-M\right)_{+}\right|^{p-2}\left(u_{m}-M\right)_{+}\right)_{T}+\left(\left(u_{m}(t),\left|\left(u_{m}-M\right)_{+}\right|^{p-2}\left(u_{m}-M\right)_{+}\right)\right)_{T} \\
& \quad+\left(g\left(u_{m}(t)\right),\left|\left(u_{m}-M\right)_{+}\right|^{p-2}\left(u_{m}-M\right)_{+}\right)_{T} \\
& \quad=\left(f_{m}(t),\left|\left(u_{m}-M\right)_{+}\right|^{p-2}\left(u_{m}-M\right)_{+}\right)_{T} .
\end{aligned}
$$

Notice

$$
\begin{aligned}
& \int_{\mathcal{O}_{T}} f_{m}(t)\left|\left(u_{m}-M\right)_{+}\right|^{p-2}\left(u_{m}-M\right)_{+} d x \\
& \quad \leq \frac{1}{\alpha_{1}} \int_{\mathcal{O}_{T}}\left|f_{m}(t)\right|^{2} d x+\frac{\alpha_{1}}{4} \int_{\mathcal{O}_{T}}\left|\left(u_{m}-M\right)_{+}\right|^{2 p-2} d x .
\end{aligned}
$$

By (1.4), as $M$ is large enough, we know $g\left(u_{m}(t)\right) \geq \frac{\alpha_{1}}{2}\left|u_{m}(t)\right|^{p-1}$, hence,

$$
\begin{aligned}
& \int_{\mathcal{O}_{T}} g\left(u_{m}(t)\right)\left|\left(u_{m}-M\right)_{+}\right|^{p-2}\left(u_{m}-M\right)_{+} d x \\
& \quad \geq \frac{\alpha_{1}}{2} \int_{\mathcal{O}_{T}}\left|u_{m}(t)\right|^{p-1}\left|\left(u_{m}-M\right)_{+}\right|^{p-2}\left(u_{m}-M\right)_{+} d x \\
& \quad \geq \frac{\alpha_{1}}{4} M^{p-2} \int_{\mathcal{O}_{T}}\left|\left(u_{m}-M\right)_{+}\right|^{p} d x+\frac{\alpha_{1}}{4} \int_{\mathcal{O}_{T}}\left|\left(u_{m}-M\right)_{+}\right|^{2 p-2} d x .
\end{aligned}
$$

Inserting (3.5) and (3.6) into (3.4), for a.e. $t \in(\tau, T)$, we deduce

$$
\frac{d}{d t}\left\|\left(u_{m}(t)-M\right)_{+}\right\|_{L^{p}\left(\mathcal{O}_{T}\right)}^{p}+\frac{\alpha_{1} p}{4} M^{p-2} \int_{\mathcal{O}_{T}}\left|\left(u_{m}(t)-M\right)_{+}\right|^{p} d x \leq \frac{p}{\alpha_{1}} \int_{\mathcal{O}_{T}}\left|f_{m}(t)\right|^{2} d x
$$

for a.e. $t \in(\tau, T)$ holds. 
Similarly, replacing $\left(u_{m}(t)-M\right)_{+}$with $\left(u_{m}(t)+M\right)_{-}$and following a similar process, we obtain

$$
\frac{d}{d t}\left\|\left(u_{m}(t)+M\right)_{-}\right\|_{L^{p}\left(\mathcal{O}_{T}\right)}^{p}+\frac{\alpha_{1} p}{4} M^{p-2} \int_{\mathcal{O}_{T}}\left|\left(u_{m}(t)+M\right)_{-}\right|^{p} d x \leq \frac{p}{\alpha_{1}} \int_{\mathcal{O}_{T}}\left|f_{m}(t)\right|^{2} d x
$$

for a.e. $t \in(\tau, T)$.

Combining inequalities (3.7) and (3.8), we see that (3.1) holds for a.e. $t \in(\tau, T)$.

The following Gronwall lemma, introduced by Łukaszewicz in [3], is useful in an inequality for estimating.

Lemma 3.2 ([3]) For some $\lambda>0, \tau \in \mathbb{R}$ and $s>\tau$, let

$$
y^{\prime}(s)+\lambda y(s) \leq h(s)
$$

where functions $y, y^{\prime}, h$ are assumed to be locally integrable and $y$, $h$ are nonnegative on the interval $t<s<t+r$, for some $t \geq \tau$. Then

$$
y(t+r) \leq e^{-\lambda \frac{r}{2}} \frac{2}{r} \int_{t}^{t+\frac{r}{2}} y(s) d s+e^{-\lambda(t+r)} \int_{t}^{t+r} e^{\lambda s} h(s) d s .
$$

In cylindrical domains, Lemma 3.3 and Lemma 3.4 in [4] have provided the method checking the conditions of asymptotic compact in $L^{p}(\mathcal{O})(p>2)$, and following a similar idea, we establish the lemma in non-cylindrical domains.

Lemma 3.3 Let $\hat{D}=\{D(\tau): \tau \in \mathbb{R}\} \in \mathscr{D}_{\lambda_{1}}$. Then, for any $\varepsilon>0$ and each $t \in \mathbb{R}$, there exist constants $M$ and $\tau_{1}=\tau_{1}(t, \hat{D})$ such that

$$
\int_{\mathcal{O}_{t}\left(\left|U(t, \tau) u_{\tau}\right| \geq M\right)}\left|\left(\left|U(t, \tau) u_{\tau}\right|-M\right)_{+}\right|^{p} d x<\varepsilon
$$

for any $\tau \leq \tau_{1}$, any $u_{\tau} \in \hat{D}$. Furthermore, there exists a constant $\bar{M}>0$ such that

$$
\int_{\mathcal{O}_{t}\left(\left|U(t, \tau) u_{\tau}\right| \geq \bar{M}\right)}\left|U(t, \tau) u_{\tau}\right|^{p} d x<\varepsilon
$$

for any $\tau \leq \tau_{1}$ and any $u_{\tau} \in \hat{D}$.

Proof Assume that $u_{\tau m} \in L^{\infty}\left(\mathcal{O}_{\tau}\right) \cap H_{0}^{1}\left(\mathcal{O}_{\tau}\right)$ and $f_{m} \in L^{\infty}\left(\tilde{Q}_{\tau, T}\right)$ for any fixed $\tau \leq T, T \in$ $\mathbb{R}$. Then for $u_{\tau} \in L^{2}\left(\mathcal{O}_{\tau}\right)$ and $f \in L_{\text {loc }}^{2}\left(\mathbb{R} ; L^{2}\left(\mathcal{O}_{t}\right)\right)$, there exist sequences $u_{\tau m} \in L^{\infty}\left(\mathcal{O}_{\tau}\right) \cap$ $H_{0}^{1}\left(\mathcal{O}_{\tau}\right), f_{m} \in L^{\infty}\left(\tilde{Q}_{\tau, T}\right)$, such that

$$
u_{\tau m} \rightarrow u_{\tau} \quad \text { in } L^{2}\left(\mathcal{O}_{\tau}\right), \quad f_{m} \rightarrow f \quad \text { in } L^{2}\left(\tau, T ; L^{2}\left(\mathcal{O}_{t}\right)\right), \quad m \rightarrow+\infty,
$$

and, for each $m=1,2, \ldots$,

$$
\left|u_{\tau m}\right|_{\tau}^{2} \leq 2\left|u_{\tau}\right|_{\tau}^{2}+1, \quad\left|f_{m}\right|_{T}^{2} \leq 2|f|_{T}^{2}+1
$$


Applying the Gronwall Lemma 3.2 to (3.1) and combining with (3.10), we obtain for $r>0$

$$
\begin{aligned}
& \left\|\left(\left|u_{m}(t+r)\right|-M\right)_{+}\right\|_{L^{p}\left(\mathcal{O}_{T}\right)}^{p} \\
& \leq e^{-\frac{\alpha_{1} p}{4} M^{p-2} \frac{r}{2}} \frac{2}{r} \int_{t}^{t+\frac{r}{2}}\left\|\left(\left|u_{m}(s)\right|-M\right)_{+}\right\|_{L^{p}\left(\mathcal{O}_{T}\right)}^{p} d s \\
& \quad+e^{-\frac{\alpha_{1} p}{4} M^{p-2}(t+r)} C_{p, \alpha_{1}, r} \int_{t}^{t+r} e^{\frac{\alpha_{1} p}{4} M^{p-2} s}|f(s)|_{T}^{2} d s \\
& \leq e^{-\frac{\alpha_{1} p}{4} M^{p-2} \frac{r}{2}} \frac{2}{r} 2^{p}\left(\int_{t}^{t+\frac{r}{2}}\left\|u_{m}(s)\right\|_{L^{p}\left(\mathcal{O}_{T}\right)}^{p} d s+\frac{r}{2} M^{p}\left|\mathcal{O}_{t+r}\right|\right) \\
& \quad+e^{-\frac{\alpha_{1} p}{4} M^{p-2}(t+r)} C_{p, \alpha_{1}, r} \int_{t}^{t+r} e^{\frac{\alpha_{1} p}{4} M^{p-2} s}|f(s)|_{T}^{2} d s .
\end{aligned}
$$

Noting $\eta u_{m} \in \mathcal{U}_{\tau, T}$ (any $\eta \in C_{c}^{1}(\tau, T)$ ), we have

$$
\begin{aligned}
& \int_{\tau}^{T}\left[-\left(u_{m}(t),\left(\eta(t) u_{m}(t)\right)^{\prime}\right)_{T}+\left(\left(u_{m}(t), \eta(t) u_{m}(t)\right)\right)_{T}+\left(g\left(u_{m}(t)\right), \eta(t) u_{m}(t)\right)_{T}\right] d t \\
& \quad=\int_{\tau}^{T}\left(f_{m}(t), \eta(t) u_{m}(t)\right)_{T} d t
\end{aligned}
$$

in addition, for a.e. $t \in(\tau, T)$,

$$
\left(u_{m}^{\prime}(t), u_{m}(t)\right)_{T}+\left(\left(u_{m}(t), u_{m}(t)\right)\right)_{T}+\left(g\left(u_{m}(t)\right), u_{m}(t)\right)_{T}=\left(f_{m}(t), u_{m}(t)\right)_{T} .
$$

By using (1.4) and the Cauchy inequality, it follows that

$$
\begin{aligned}
& \frac{d}{d t}\left|u_{m}(t)\right|_{T}^{2}+\left\|u_{m}(t)\right\|_{T}^{2}+2 \alpha_{1}\left\|u_{m}(t)\right\|_{L^{p}\left(\mathcal{O}_{T}\right)}^{p} \\
& \quad \leq \frac{1}{\lambda_{1, T}}\left|f_{m}(t)\right|_{T}^{2}+2 \beta\left|\mathcal{O}_{T}\right| \quad \text { a.e. } t \in(\tau, T),
\end{aligned}
$$

and, in particular, that

$$
\begin{aligned}
& \frac{d}{d t}\left|u_{m}(t)\right|_{T}^{2}+\lambda_{1, T}\left|u_{m}(t)\right|_{T}^{2}+2 \alpha_{1}\left\|u_{m}(t)\right\|_{L^{p}\left(\mathcal{O}_{T}\right)}^{p} \\
& \leq \frac{1}{\lambda_{1, T}}\left|f_{m}(t)\right|_{T}^{2}+2 \beta\left|\mathcal{O}_{T}\right| \quad \text { a.e. } t \in(\tau, T) .
\end{aligned}
$$

Integrating the inequality (3.13) over $\left[t, t+\frac{r}{2}\right]$, we have

$$
\begin{aligned}
& 2 \alpha_{1} \int_{t}^{t+\frac{r}{2}}\left\|u_{m}(s)\right\|_{L^{p}\left(\mathcal{O}_{T}\right)}^{p} d s \\
& \quad \leq \frac{1}{\lambda_{1, T}} \int_{t}^{t+\frac{r}{2}}\left|f_{m}(s)\right|_{T}^{2} d s+r \beta\left|\mathcal{O}_{T}\right|+\left|u_{m}(t)\right|_{T}^{2} \\
& \quad \leq \frac{2}{\lambda_{1, T}} \int_{t}^{t+\frac{r}{2}}|f(s)|_{T}^{2} d s+\frac{r}{2 \lambda_{1, T}}+r \beta\left|\mathcal{O}_{T}\right|+\left|u_{m}(t)\right|_{T}^{2}
\end{aligned}
$$


for any $t \in(\tau, T)$. In addition, applying the Gronwall lemma to (3.13), we also have

$$
\begin{aligned}
\left|u_{m}(t)\right|_{T}^{2} \leq & e^{-\lambda_{1, T}(t-\tau)}\left|u_{\tau m}\right|_{\tau}^{2}+\frac{1}{\lambda_{1, T}} e^{-\lambda_{1, T} t} \int_{\tau}^{t} e^{\lambda_{1, T} s}\left|f_{m}(s)\right|_{T}^{2} d s+\frac{2 \beta\left|\mathcal{O}_{T}\right|}{\lambda_{1, T}} \\
\leq & e^{-\lambda_{1, T}(t-\tau)}\left(2\left|u_{\tau}\right|_{\tau}^{2}+1\right)+C \frac{1}{\lambda_{1, T}} e^{-\lambda_{1, T} t} \int_{-\infty}^{t} e^{\lambda_{1, T} s}|f(s)|_{T}^{2} d s \\
& +C \frac{1}{\lambda_{1, T}} e^{-\lambda_{1, T} t}+\frac{2 \beta\left|\mathcal{O}_{T}\right|}{\lambda_{1, T}}
\end{aligned}
$$

for any $t \in[\tau, T]$, where $\lambda_{1, T}$ is the first eigenvalue of $-\Delta$ in $H_{0}^{1}\left(\mathcal{O}_{T}\right)$.

Combining (3.14), (3.15), and (3.11), we know that

$$
\begin{aligned}
& \left\|\left(\left|u_{m}(t+r)\right|-M\right)_{+}\right\|_{L^{p}\left(\mathcal{O}_{T}\right)}^{p} \\
& \quad \leq e^{-\frac{\alpha_{1} p}{4} M^{p-2} \frac{r}{2}} \frac{2}{r} 2^{p} C_{1}+e^{-\frac{\alpha_{1} p}{4} M^{p-2}(t+r)} C_{p, \alpha_{1}, r} \int_{t}^{t+r} e^{\frac{\alpha_{1} p}{4} M^{p-2} s}|f(s)|_{T}^{2} d s,
\end{aligned}
$$

where $C_{1}$ is a constant independent of $m$, and depending on $\frac{1}{\alpha_{1}}, e^{-\lambda_{1, T}(t-\tau)}\left|u_{\tau}\right|_{\tau}^{2}$, $\int_{-\infty}^{t} e^{\lambda_{1}, T s}|f(s)|_{T}^{2} d s, \frac{2 \beta\left|\mathcal{O}_{T}\right|}{\lambda_{1, T}}, \int_{t}^{t+\frac{r}{2}}|f(s)|_{T}^{2} d s$.

So, $\left(\left|u_{m}(t+r)\right|-M\right)_{+}$is bounded in $L^{\infty}\left(\tau, T ; L^{p}\left(\mathcal{O}_{T}\right)\right)$ and there exists a subsequence denoted still by $\left(\left|u_{m}(t+r)\right|-M\right)_{+}$such that

$$
\left(\left|u_{m}\right|-M\right)_{+} \rightarrow(|u|-M)_{+} \quad \text { weakly star convergence in } L^{\infty}\left(\tau, T ; L^{p}\left(\mathcal{O}_{T}\right)\right) .
$$

Hence,

$$
\begin{aligned}
& \left\|(|u(t+r)|-M)_{+}\right\|_{L^{p}\left(\mathcal{O}_{T}\right)}^{p} \\
& \leq e^{-\frac{\alpha_{1} p}{4} M^{p-2} \frac{r}{2}} \frac{2}{r} 2^{p} C_{1}+e^{-\frac{\alpha_{1} p}{4} M^{p-2}(t+r)} C_{p, \alpha_{1}, r} \int_{t}^{t+r} e^{\frac{\alpha_{1} p}{4} M^{p-2} s}|f(s)|_{T}^{2} d s
\end{aligned}
$$

for the $C_{1}$ above.

For any $t \in \mathbb{R}, \varepsilon>0$, and any $\hat{\mathcal{D}} \in \mathscr{D}_{\lambda_{1}}$, there exist constants $\tau_{1}(t, \hat{\mathcal{D}})$ and $M_{1}$ such that for $M \geq M_{1}$ and $\tau<\tau_{1}(t, \hat{\mathcal{D}})$, one obtains

$$
e^{-\frac{\alpha_{1} p}{4} M^{p-2} \frac{r}{2}} \frac{2}{r} 2^{p} C_{1}<\frac{\varepsilon}{2}
$$

moreover, with $M$ large enough and the function $f$ integrable on $[t, t+r]$, we have

$$
e^{-\frac{\alpha_{1} p}{4} M^{p-2}(t+r)} \frac{2 p}{r \alpha_{1}} \int_{t}^{t+r} e^{\frac{\alpha_{1} p}{4} M^{p-2} s}|f(s)|_{T}^{2} d s<\frac{\varepsilon}{2}
$$

Therefore,

$$
\left\|(|u(t+r)|-M)_{+}\right\|_{L^{p}\left(\mathcal{O}_{T}\right)}^{p}<\varepsilon
$$

for $M \geq M_{1}$ and $\tau<\tau_{1}(t, \hat{\mathcal{D}})$. 
Finally, let $\bar{M}:=2 M$, and by (3.9) we conclude that

$$
\begin{aligned}
& \int_{\mathcal{O}_{t}\left(\left|U(t, \tau) u_{\tau}\right| \geq 2 M\right)}\left|U(t, \tau) u_{\tau}\right|^{p} d x \\
& =\int_{\left.\mathcal{O}_{t}|| U(t, \tau) u_{\tau} \mid \geq 2 M\right)}\left(\left(\left|U(t, \tau) u_{\tau}\right|-M\right)+M\right)^{p} d x \\
& \quad \leq 2^{p-1}\left(\int_{\mathcal{O}_{t}\left(\left|U(t, \tau) u_{\tau}\right| \geq 2 M\right)}\left(\left|U(t, \tau) u_{\tau}\right|-M\right)^{p} d x+\int_{\mathcal{O}_{t}\left(\left|U(t, \tau) u_{\tau}\right| \geq 2 M\right)} M^{p} d x\right) \\
& \quad \leq 2^{p-1}\left(\int_{\mathcal{O}_{t}\left(\left|U(t, \tau) u_{\tau}\right| \geq M\right)}\left(\left|U(t, \tau) u_{\tau}\right|-M\right)^{p} d x\right. \\
& \left.\quad+\int_{\mathcal{O}_{t}\left(\left|U(t, \tau) u_{\tau}\right| \geq M\right)}\left(\left|U(t, \tau) u_{\tau}\right|-M\right)^{p} d x\right) \\
& \leq 2^{p} \varepsilon
\end{aligned}
$$

for any $\tau \leq \tau_{1}(t, \hat{\mathcal{D}})$ and any $u_{\tau} \in \hat{D}$.

Now, we prove Theorem 1.1.

According to Lemma 2.10, Theorem 2.5, and Lemma 3.3, it is sufficient to check the existence of a pullback $\mathscr{D}_{\lambda_{1}}$ absorbing set in $L^{p}$.

By Lemma 3.3, for any $u_{\tau} \in \hat{D}$ and any $\tau \leq \tau_{1}(t, \varepsilon, \hat{D})$, we have

$$
\begin{aligned}
& \int_{\mathcal{O}_{t}}\left|U(t, \tau) u_{\tau}\right|^{p} d x \\
& \quad=\int_{\mathcal{O}_{t}\left(\left|U(t, \tau) u_{\tau}\right| \leq 2 M\right)}\left|U(t, \tau) u_{\tau}\right|^{p} d x+\int_{\mathcal{O}_{t}\left(\left|U(t, \tau) u_{\tau}\right| \geq 2 M\right)}\left|U(t, \tau) u_{\tau}\right|^{p} d x \\
& \quad \leq(2 M)^{p}\left|\mathcal{O}_{t}\right|+2^{p} \int_{\mathcal{O}_{t}\left(\left|U(t, \tau) u_{\tau}\right| \geq M\right)}\left(\left|U(t, \tau) u_{\tau}\right|-M\right)_{+}^{p} d x \\
& \quad \leq(2 M)^{p}\left|\mathcal{O}_{t}\right|+1 .
\end{aligned}
$$

\section{Pullback $\mathscr{D}_{\lambda_{1}}$ attractor in $L^{2+\delta}$}

Throughout this section, the $T$ will be required to obey $T \geq t$ or $T \geq s+1$. We first consider the higher-order integrability for approximation solutions and variational solutions of (1.6), respectively, then we obtain a pullback $\mathscr{D}_{\lambda_{1}}$ absorbing set in $L^{2+\delta}\left(\mathcal{O}_{t}\right)(\forall \delta \in[0, \infty))$. Finally, we prove the existence of a pullback $\mathscr{D}_{\lambda_{1}}$ attractor in $L^{2+\delta}\left(\mathcal{O}_{t}\right)$ for any $\delta \in[0, \infty)$.

\subsection{Higher-order integrability for approximation solutions}

For any $\hat{D}=\{D(t): t \in \mathbb{R}\} \in \mathscr{D}_{\lambda_{1}}$ and $u_{\tau} \in D(\tau)$, set $u(t)=U(t, \tau) u_{\tau}$. For any fixed $\tau \in$ $(-\infty, T]$, denoted by $u_{m}(t)$, we have the variational solution of equation (1.6) corresponding to the data $\left(u_{\tau m}, f\right)$ satisfying

$$
u_{\tau m} \in L^{\infty}\left(\mathcal{O}_{\tau}\right) \cap H_{0}^{1}\left(\mathcal{O}_{\tau}\right) \text { and } f \in L^{\infty}\left(\tilde{Q}_{\tau, T}\right)
$$

By Lemma 2.12 and Theorem 2.8, we know

$$
u_{m} \in L^{2}\left(\tau, T ; H_{0}^{1}\left(\mathcal{O}_{T}\right)\right) \cap L^{\infty}\left(\tilde{Q}_{\tau, T}\right)
$$


and

$$
\int_{\tau}^{T}\left[-\left(u_{m}(t), \phi^{\prime}(t)\right)_{T}+\left(\left(u_{m}(t), \phi(t)\right)\right)_{T}+\left(g\left(u_{m}(t)\right), \phi(t)\right)_{T}\right] d t=\int_{\tau}^{T}(f(t), \phi)_{T} d t
$$

for any $\phi \in \mathcal{U}_{\tau, T}$.

For any $\theta \geq 0$, we still have

$$
\left|u_{m}\right|^{\theta} u_{m} \in L^{2}\left(\tau, T ; H_{0}^{1}\left(\mathcal{O}_{T}\right)\right) \cap L^{\infty}\left(\tilde{Q}_{\tau, T}\right),
$$

and, for any $\eta \in C_{c}^{1}(\tau, T)$,

$$
\begin{aligned}
& \eta\left|u_{m}\right|^{\theta} u_{m} \in L^{2}\left(\tau, T ; H_{0}^{1}\left(\mathcal{O}_{T}\right)\right) \cap L^{\infty}\left(\tilde{Q}_{\tau, T}\right), \\
& \frac{d}{d t}\left(\eta\left|u_{m}\right|^{\theta} u_{m}\right) \in L^{2}\left(\tau, T ; L^{2}\left(\mathcal{O}_{T}\right)\right), \\
& \eta(T)\left|u_{m}(T)\right|^{\theta} u_{m}(T)=\eta(\tau)\left|u_{m}(\tau)\right|^{\theta} u_{m}(\tau)=0, \\
& \eta(t)\left|u_{m}(t)\right|^{\theta} u_{m}(t) \in H_{0}^{1}\left(\mathcal{O}_{t}\right) \quad \text { a.e. } t \in(\tau, T) .
\end{aligned}
$$

Hence, we can choose $\eta\left|u_{m}\right|^{\theta} u_{m}$ as a test function to obtain

$$
\begin{aligned}
\int_{\tau}^{T}[ & -\left(u_{m}(t),\left(\eta(t)\left|u_{m}(t)\right|^{\theta} u_{m}(t)\right)^{\prime}\right)_{T}+\left(\left(u_{m}(t), \eta(t)\left|u_{m}(t)\right|^{\theta} u_{m}(t)\right)\right)_{T} \\
& \left.+\left(g\left(u_{m}(t)\right), \eta(t)\left|u_{m}(t)\right|^{\theta} u_{m}(t)\right)_{T}\right] d t \\
= & \int_{\tau}^{T}\left(f(t), \eta(t)\left|u_{m}(t)\right|^{\theta} u_{m}(t)\right)_{T} d t
\end{aligned}
$$

moreover,

$$
\begin{aligned}
& \int_{\tau}^{T}\left[\left(u_{m}^{\prime}(t),\left|u_{m}(t)\right|^{\theta} u_{m}(t)\right)_{T}+\left(\left(u_{m}(t),\left|u_{m}(t)\right|^{\theta} u_{m}(t)\right)\right)_{T}\right. \\
& \left.\quad+\left(g\left(u_{m}(t)\right),\left|u_{m}(t)\right|^{\theta} u_{m}(t)\right)_{T}-\left(f(t),\left|u_{m}(t)\right|^{\theta} u_{m}(t)\right)_{T}\right] \eta(t) d t=0, \\
& \quad \text { for any } \eta \in C_{c}^{1}(\tau, T) .
\end{aligned}
$$

Therefore, for a.e. $t \in(\tau, T)$,

$$
\begin{aligned}
& \left(u_{m}^{\prime}(t),\left|u_{m}(t)\right|^{\theta} u_{m}(t)\right)_{T}+\left(\left(u_{m}(t),\left|u_{m}(t)\right|^{\theta} u_{m}(t)\right)\right)_{T}+\left(g\left(u_{m}(t)\right),\left|u_{m}(t)\right|^{\theta} u_{m}(t)\right)_{T} \\
& \quad=\left(f(t),\left|u_{m}(t)\right|^{\theta} u_{m}(t)\right)_{T} .
\end{aligned}
$$

By (1.4), we know

$$
\int_{\mathcal{O}_{T}} g\left(u_{m}(t)\right)\left|u_{m}(t)\right|^{\theta} u_{m}(t) d x \geq-\beta \int_{\mathcal{O}_{T}}\left|u_{m}(t)\right|^{\theta} d x+\alpha_{1} \int_{\mathcal{O}_{T}}\left|u_{m}(t)\right|^{\theta+p} d x
$$

Noticing (3.3), we can select an appropriate $K^{\prime}$ such that

$$
u_{m}(t) \geq M>1 \quad \text { for a.e. } t \in(\tau, T) .
$$


So,

$$
\begin{aligned}
\int_{\mathcal{O}_{T}} f(t)\left|u_{m}(t)\right|^{\theta} u_{m}(t) d x & \leq \int_{\mathcal{O}_{T}}\left|f(t) \| u_{m}(t)\right|^{\theta+1} d x \\
& \leq\|f(t)\|_{L^{\infty}\left(\mathcal{O}_{T}\right)} \int_{\mathcal{O}_{T}}\left|u_{m}(t)\right|^{\theta+1} d x \\
& \leq\|f(t)\|_{L^{\infty}\left(\mathcal{O}_{T}\right)} \int_{\mathcal{O}_{T}}\left|u_{m}(t)\right|^{\theta+2} d x .
\end{aligned}
$$

Combining (4.2), (4.3), and (4.5), we deduce

$$
\begin{aligned}
& \frac{1}{\theta+2} \frac{d}{d t}\left\|u_{m}(t)\right\|_{L^{\theta+2}\left(\mathcal{O}_{T}\right)}^{\theta+2}-\frac{1}{\theta+2} \int_{\partial \mathcal{O}_{T}}\left|\gamma u_{m}(t)\right|^{\theta+2} d S \\
& \quad+\left.\left.(\theta+1)\left(\frac{2}{\theta+2}\right)^{2} \int_{\mathcal{O}_{T}}|\nabla| u_{m}(t)\right|^{1+\frac{\theta}{2}}\right|^{2} d x+\alpha_{1}\left\|u_{m}(t)\right\|_{L^{p+\theta}\left(\mathcal{O}_{T}\right)}^{p+\theta} \\
& \leq \beta\left\|u_{m}(t)\right\|_{L^{\theta}\left(\mathcal{O}_{T}\right)}^{\theta}+\|f(t)\|_{L^{\infty}\left(\mathcal{O}_{T}\right)}\left\|u_{m}(t)\right\|_{L^{\theta+2}\left(\mathcal{O}_{T}\right)}^{\theta+2}
\end{aligned}
$$

for a.e. $t \in(\tau, T)$. Due to $u_{m}(t) \in H_{0}^{1}\left(\mathcal{O}_{t}\right)$ and

$$
\left\|u_{m}(t)\right\|_{L^{\theta}\left(\mathcal{O}_{T}\right)}^{\theta} \leq\left|\mathcal{O}_{T}\right|^{\frac{2}{\theta+2}}\left\|u_{m}(t)\right\|_{L^{\theta+2}\left(\mathcal{O}_{T}\right)}^{\theta+2},
$$

it follows from (4.6) that

$$
\begin{aligned}
& \frac{1}{\theta+2} \frac{d}{d t}\left\|u_{m}(t)\right\|_{L^{\theta+2}\left(\mathcal{O}_{T}\right)}^{\theta+2}+\left.\left.(\theta+1)\left(\frac{2}{\theta+2}\right)^{2} \int_{\mathcal{O}_{T}}|\nabla| u_{m}(t)\right|^{1+\frac{\theta}{2}}\right|^{2} d x+\alpha_{1}\left\|u_{m}(t)\right\|_{L^{p+\theta}\left(\mathcal{O}_{T}\right)}^{p+\theta} \\
& \quad \leq C_{\beta,\left|\mathcal{O}_{T}\right|, \theta,\|f(t)\|_{L^{\infty}\left(\mathcal{O}_{T}\right)}\left\|u_{m}(t)\right\|_{L^{\theta+2}\left(\mathcal{O}_{T}\right)}^{\theta+2}} \text { for a.e. } t \in(\tau, T) .
\end{aligned}
$$

We prove the following result.

Theorem 4.1 Let $\hat{D} \in \mathscr{D}_{\lambda_{1}}$ and $T$ be a fixed time. Assume further that the initial data $u_{\tau m}$ and $f$ satisfy (4.1). Then, for each $t \in(\tau, T)$ and each $h=0,1,2, \ldots$, there exist two sequences (positive constants) $\tilde{T}_{h}(t, \hat{D})$ (depending only on $h, t, \lambda_{1, T}$ and $\hat{D}$ ) and $\tilde{M}_{h}(t)$ (depending only on $t, h, \lambda_{1, T}, N,\left|\mathcal{O}_{T}\right|$ and $\left.\|f(t)\|_{L^{\infty}\left(\mathcal{O}_{T}\right)}\right)$, such that for any $m=1,2, \ldots$, the variational solution $u_{m}$ of (1.6) satisfies

$$
\left(A_{h}\right) \quad \int_{\mathcal{O}_{T}}\left|u_{m}(t)\right|^{2\left(\frac{N}{N-2}\right)^{h}} d x \leq \tilde{M}_{h}(t) \quad \text { for any } t-\tau \geq \tilde{T}_{h}(t, \hat{D})
$$

and

$$
\left(B_{h}\right) \quad \int_{s}^{s+1}\left(\int_{\mathcal{O}_{T}}\left|u_{m}(r, x)\right|^{2\left(\frac{N}{N-2}\right)^{h+1}} d x\right)^{\frac{N-2}{N}} d r \leq \tilde{M}_{h}(t)
$$

for any $s-\tau \geq \tilde{T}_{h}(t, \hat{D})$.

Proof Selecting $\theta=0$ in (4.2), for a.e. $t \in(\tau, T)$, we have

$$
\left(u_{m}^{\prime}(t), u_{m}(t)\right)_{T}+\left(\left(u_{m}(t), u_{m}(t)\right)\right)_{T}+\left(g\left(u_{m}(t)\right), u_{m}(t)\right)_{T}=\left(f(t), u_{m}(t)\right)_{T} .
$$


By using (1.4) and the Cauchy inequality, it follows that

$$
\frac{d}{d t}\left|u_{m}(t)\right|_{T}^{2}+\left\|u_{m}(t)\right\|_{T}^{2}+2 \alpha_{1}\left\|u_{m}(t)\right\|_{L^{p}\left(\mathcal{O}_{T}\right)}^{p} \leq \frac{1}{\lambda_{1, T}}|f(t)|_{T}^{2}+2 \beta\left|\mathcal{O}_{T}\right| \quad \text { a.e. } t \in(\tau, T),
$$

and, in particular, that

$$
\begin{gathered}
\frac{d}{d t}\left|u_{m}(t)\right|_{T}^{2}+\lambda_{1, T}\left|u_{m}(t)\right|_{T}^{2}+2 \alpha_{1}\left\|u_{m}(t)\right\|_{L^{p}\left(\mathcal{O}_{T}\right)}^{p} \\
\leq \frac{1}{\lambda_{1, T}}|f(t)|_{T}^{2}+2 \beta\left|\mathcal{O}_{T}\right| \quad \text { a.e. } t \in(\tau, T)
\end{gathered}
$$

moreover,

$$
\left|u_{m}(t)\right|_{T}^{2} \leq e^{-\lambda_{1, T}(t-\tau)}\left|u_{\tau m}\right|_{\tau}^{2}+\frac{\left|\mathcal{O}_{T}\right|\|f(t)\|_{L^{\infty}\left(\mathcal{O}_{T}\right)}^{2}}{\lambda_{1, T}^{2}}+\frac{2 \beta\left|\mathcal{O}_{T}\right|}{\lambda_{1, T}}
$$

for any $t \in[\tau, T]$, where $\lambda_{1, T}$ is the first eigenvalue of $-\Delta$ in $H_{0}^{1}\left(\mathcal{O}_{T}\right)$.

By (3.10) and (4.10), we know

$$
\begin{aligned}
\left|u_{m}(t)\right|_{T}^{2} \leq & e^{-\lambda_{1, T}(t-\tau)}\left(2\left|u_{\tau}\right|_{\tau}^{2}+1\right) \\
& +\frac{\left|\mathcal{O}_{T}\right| \mid f(t) \|_{L^{\infty}\left(\mathcal{O}_{T}\right)}^{2}}{\lambda_{1, T}^{2}}+\frac{2 \beta\left|\mathcal{O}_{T}\right|}{\lambda_{1, T}} \quad \text { for all } t \in(\tau, T) .
\end{aligned}
$$

For each $t \in \mathbb{R}$, let

$$
\tilde{M}_{0}^{\prime}(t):=\frac{\left|\mathcal{O}_{T}\right|\|f(t)\|_{L^{\infty}\left(\mathcal{O}_{T}\right)}^{2}}{\lambda_{1, T}^{2}}+\frac{2 \beta\left|\mathcal{O}_{T}\right|}{\lambda_{1, T}}
$$

combined with (4.11) to get

$$
\left|u_{m}(t)\right|_{T}^{2} \leq e^{-\lambda_{1, T}(t-\tau)}\left(2\left|u_{\tau}\right|_{\tau}^{2}+1\right)+\tilde{M}_{0}^{\prime}(t)
$$

Noticing that $u_{\tau} \in D(\tau)$ with $\hat{D}=\{D(t): t \in \mathbb{R}\} \in \mathscr{D}_{\lambda_{1}}$, by (4.12), there exists a constant $T^{\prime}(t, \hat{D})$ such that

$$
\left|u_{m}(t)\right|_{T}^{2} \leq 1+\tilde{M}_{0}^{\prime}(t) \quad \text { for all } t-\tau \geq T^{\prime}(t, \hat{D})
$$

Taking $\theta=0$ in (4.7) and integrating with respect to $t$, we obtain

$$
\begin{aligned}
& 2 \int_{s}^{s+1} \int_{\mathcal{O}_{T}}|\nabla| u_{m}(t)||^{2} d x d t \leq\left(C_{\beta,\left|\mathcal{O}_{T}\right|,\|f(t)\|_{L}{ }^{\infty}\left(\mathcal{O}_{T}\right)}+1\right)\left(\tilde{M}_{0}^{\prime}(t)+1\right) \\
& \quad \text { for all } s-\tau \geq T^{\prime}(t, \hat{D}) .
\end{aligned}
$$

On the other hand, from the embedding $H_{0}^{1}\left(\mathcal{O}_{T}\right) \hookrightarrow L^{\frac{2 N}{N-2}}\left(\mathcal{O}_{T}\right)$, we know that there is a constant $c_{N}$ such that

$$
\|\phi\|_{L \frac{2 N}{N-2}\left(\mathcal{O}_{T}\right)} \leq c_{N}|\nabla| \phi||_{L^{2}\left(\mathcal{O}_{T}\right)} \quad \forall \phi \in H_{0}^{1}\left(\mathcal{O}_{T}\right) .
$$


From (4.14) and (4.15), it follows that

$$
\begin{aligned}
& 2 \int_{s}^{s+1}\left\|u_{m}(t)\right\|_{L^{\frac{2 N}{N-2}}\left(\mathcal{O}_{T}\right)}^{2} d t \leq c_{N}^{2}\left(C_{\beta,\left|\mathcal{O}_{T}\right|,\|f(t)\|_{L}{ }_{\left(\mathcal{O}_{T}\right)}}+1\right)\left(\tilde{M}_{0}^{\prime}(t)+1\right) \\
& \quad \text { for all } s-\tau \geq T^{\prime}(t, \hat{D}) .
\end{aligned}
$$

Setting

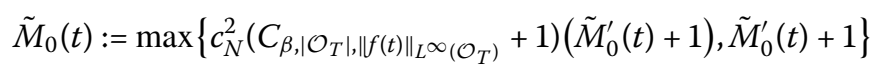

and by (4.13), (4.16), we know that $\left(A_{0}\right)$ and $\left(B_{0}\right)$ hold.

In the following, performing induction, we assume $\left(A_{h}\right)$ and $\left(B_{h}\right)$ hold to prove $\left(A_{h+1}\right)$ and $\left(B_{h+1}\right)$.

Taking $\theta=2\left(\frac{N}{N-2}\right)^{h+1}-2$ in $(4.7)$, we obtain

$$
\begin{aligned}
& \frac{d}{d t}\left\|u_{m}(t)\right\|_{L^{2\left(\frac{N}{N-2}\right)^{h+1}\left(\mathcal{O}_{T}\right)}}^{2\left(\frac{N}{h+1}\right.}+\left.\left.\left(4\left(\frac{N}{N-2}\right)^{h+1}-2\right)\left(\frac{N-2}{N}\right)^{h+1} \int_{\mathcal{O}_{T}}|\nabla| u_{m}(t)\right|^{\left(\frac{N}{N-2}\right)^{h+1}}\right|^{2} d x \\
& +2 \alpha_{1}\left(\frac{N}{N-2}\right)^{h+1}\left\|u_{m}(t)\right\|_{L^{p+2\left(\frac{N}{N-2}\right)^{h+1}-2}\left(\mathcal{O}_{T}\right)}^{p+2\left(\frac{N}{N-2}\right)^{h+1}-2} \\
& \leq C_{\beta,\left|\mathcal{O}_{T}\right|, N, h,\|f(t)\|_{L} \infty\left(\mathcal{O}_{T}\right)}\left\|u_{m}(t)\right\|_{L^{2\left(\frac{N}{N-2}\right)^{h+1}\left(\mathcal{O}_{T}\right)}}^{2\left(\frac{N}{N-2}\right)^{h+1}} \quad \text { a.e. } t \in(\tau, T),
\end{aligned}
$$

moreover,

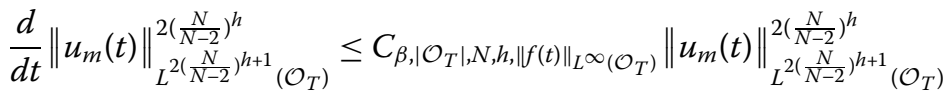

$$
\begin{aligned}
& \text { a.e. } t \in(\tau, T) \text {. }
\end{aligned}
$$

Applying the uniform Gronwall lemma to (4.18) and by $\left(B_{h}\right)$ we get

$$
\begin{aligned}
& \int_{\mathcal{O}_{T}}\left|u_{m}(t)\right|^{2\left(\frac{N}{N-2}\right)^{h+1}} d x \leq C_{\tilde{M}_{h}(t), \beta,\left|\mathcal{O}_{T}\right|, N, h,\|f(t)\|_{L}{ }^{\infty}\left(\mathcal{O}_{T}\right)} \\
& \quad \text { for any } t-\tau \geq \tilde{T}_{h}(t, \hat{D})+1
\end{aligned}
$$

For any $s-\tau \geq \tilde{T}_{h}(t, \hat{D})+1$, we integrate (4.17) over $[s, s+1]$ and obtain

$$
\left.\left.\int_{s}^{s+1} \int_{\mathcal{O}_{T}}|\nabla| u_{m}(x, t)\right|^{\left(\frac{N}{N-2}\right)^{h+1}}\right|^{2} d x d t \leq C_{\tilde{M}_{h}(t), \beta,\left|\mathcal{O}_{T}\right|, N, h,\|f(t)\|_{L}{ }^{\infty}\left(\mathcal{O}_{T}\right)}^{\prime} .
$$

Since $u_{m}$ is a variational solution of (1.6) and bounded in $L^{\infty}\left(\tilde{Q}_{\tau, T}\right), u_{m} \in H_{0}^{1}\left(\mathcal{O}_{t}\right) \cap L^{\infty}\left(\mathcal{O}_{t}\right)$ for a.e. $t \in(\tau, T)$, and $\left|u_{m}(t)\right|^{\left(\frac{N}{N-2}\right)^{h+1}} \in H_{0}^{1}\left(\mathcal{O}_{t}\right)$ for a.e. $t \in(\tau, T)$, again with the embedding

$$
\left\|\left|u_{m}(t)\right|^{\left(\frac{N}{N-2}\right)^{h+1}}\right\|_{L \frac{2 N}{N-2}\left(\mathcal{O}_{T}\right)} \leq\left.\left. c_{N}|\nabla| u_{m}(t)\right|^{\left(\frac{N}{N-2}\right)^{h+1}}\right|_{L^{2}\left(\mathcal{O}_{T}\right)}
$$

to get

$$
\int_{s}^{s+1}\left(\int_{\mathcal{O}_{T}}\left|u_{m}(t)\right|^{2\left(\frac{N}{N-2}\right)^{h+2}} d x\right)^{\frac{N-2}{N}} d t \leq c_{N}^{2} C_{\left.\tilde{M}_{h}(t), \beta,\left|\mathcal{O}_{T}\right|, N, h,\|f(t)\|_{L} \infty_{(\mathcal{O}}\right)}^{\prime}
$$


for any $s-\tau \geq \tilde{T}_{h}(t, \hat{D})+1$. Therefore, we set

$$
\tilde{T}_{h+1}(t, \hat{D})=\tilde{T}_{h}(t, \hat{D})+1
$$

and

$$
\tilde{M}_{h+1}(t)=\max \left\{C_{\tilde{M}_{h}(t), \beta,\left|\mathcal{O}_{T}\right|, N, h,\|f(t)\|_{L} \infty\left(\mathcal{O}_{T}\right)}, c_{N}^{2} C_{\tilde{M}_{h}(t), \beta,\left|\mathcal{O}_{T}\right|, N, h,\|f(t)\|_{L} \infty_{\left(\mathcal{O}_{T}\right)}}^{\prime}\right\},
$$

from (4.19) and (4.21) we know that $\left(A_{h+1}\right)$ and $\left(B_{h+1}\right)$ hold.

\subsection{Higher-order integrability for variational solutions}

Now, as initial data $u_{\tau} \in L^{2}\left(\mathcal{O}_{\tau}\right)$, we establish the following results for the variational solution of equation (1.3).

Theorem 4.2 Let $\hat{D}=\{D(\tau): \tau \in \mathbb{R}\} \in \mathscr{D}_{\lambda_{1}}$. Then for each $t \in \mathbb{R}$ and each $\delta \in[0, \infty)$, there exist constants $T(t, \delta, \hat{D})$ and $M_{\delta}(t)$ such that

$$
\|u(t)\|_{L^{2+\delta}\left(\mathcal{O}_{t}\right)}<M_{\delta}(t) \quad \text { for any } t-\tau \geq T(t, \delta, \hat{D}) .
$$

Proof For each fixed $t \in \mathbb{R}$ and $h \in\{0,1,2, \ldots\}$, take

$$
T_{h}(t, \hat{D})=\tilde{T}_{h}(t, \hat{D})+1,
$$

where $\tilde{T}_{h}(t, \hat{D})$ is just the constant given in Theorem 4.1 corresponding to the pair $t, h$.

Setting

$$
T=t+1
$$

and for any (fixed) $\tau$ satisfying

$$
\tau \leq t-T_{h}(t, \hat{D})
$$

we consider the approximation on the interval $[\tau, T]$.

When $u_{\tau} \in L^{2}\left(\mathcal{O}_{\tau}\right)$, there exists a sequence $\left\{u_{\tau m}\right\} \subset L^{\infty}\left(\mathcal{O}_{\tau}\right)$ such that

$$
u_{\tau m} \rightarrow u_{\tau} \text { in } L^{2}\left(\mathcal{O}_{\tau}\right) \quad \text { as } m \rightarrow \infty .
$$

On account of the proof of Proposition 11 in [10], we have

$$
\begin{gathered}
\left|u_{m}(t)-u_{n}(t)\right|_{T}^{2}+2 \int_{\tau}^{t}\left\|u_{m}(s)-u_{n}(s)\right\|_{T}^{2} d s \\
\quad \leq\left|u_{\tau m}-u_{\tau n}\right|_{T}^{2}+2 l \int_{\tau}^{t}\left|u_{m}(s)-u_{n}(s)\right|_{T}^{2} d s
\end{gathered}
$$

for all $t \in[\tau, T]$ and $n, m \geq 1$. By applying the Gronwall lemma, we conclude from the inequality above that

$$
\left\{u_{m}\right\} \text { is a Cauchy sequence in } L^{2}\left(\tau, T ; H_{0}^{1}\left(\mathcal{O}_{T}\right)\right) \cap C\left([\tau, T] ; L^{2}\left(\mathcal{O}_{T}\right)\right) .
$$


On the other hand, by (4.9), it can be proved that

$$
\left\{u_{m}\right\} \text { is bounded in } L^{\infty}\left(\tau, T ; L^{2}\left(\mathcal{O}_{T}\right)\right) \cap L^{2}\left(\tau, T ; H_{0}^{1}\left(\mathcal{O}_{T}\right)\right) .
$$

Consequently, from (4.23) and (4.24), it follows that

$$
u_{m} \rightarrow u \quad \text { in } C\left([\tau, T] ; L^{2}\left(\mathcal{O}_{T}\right)\right)
$$

in particular, for each $t \in[\tau, T]$, there exists a subsequence $\left\{u_{m j}\right\}$ such that

$$
u_{m j}(t) \rightarrow u(t) \quad \text { a.e. } x \in \mathcal{O}_{T}
$$

Therefore, set $\bar{M}_{h}(t)=\tilde{M}_{h}(t)$ for $\left(A_{h}\right)$ and $\left(B_{h}\right)$ in Theorem 4.1, and as $m \rightarrow+\infty$, according to Fatou's lemma:

$$
\begin{aligned}
& \int_{\mathcal{O}_{T}}\left|U(t, \tau) u_{\tau}\right|^{2\left(\frac{N}{N-2}\right)^{h}} d x \\
& \quad \leq \liminf _{m \rightarrow \infty} \int_{\mathcal{O}_{T}}\left|u_{m j}(t)\right|^{2\left(\frac{N}{N-2}\right)^{h}} d x \\
& \leq \bar{M}_{h}(t)
\end{aligned}
$$

for any $t-\tau \geq T_{h}(t, \hat{D})$ holds.

For each $\delta \in[0, \infty)$, there is a unique $h \in\{1,2,3, \ldots\}$ such that

$$
2+\delta+1 \in\left(2\left(\frac{N}{N-2}\right)^{h-1}, 2\left(\frac{N}{N-2}\right)^{h}\right]
$$

Then, for each $\delta \in[0, \infty)$ and anyt $-\tau \geq T_{h}(t, \hat{D})$, we have

$$
\begin{aligned}
\|u(t)\|_{L^{2+\delta}\left(\mathcal{O}_{t}\right)}^{2+\delta} & \leq\|u(t)\|_{L^{2\left(\frac{N}{N-2}\right)^{h}}\left(\mathcal{O}_{t}\right)}^{\frac{2+\delta}{2\left(\frac{N}{N^{h}}\right.}} \cdot\left|\mathcal{O}_{t}\right|^{1-\frac{2+\delta}{2\left(\frac{N}{N-2}\right)^{h}}} \\
& \leq\left(\bar{M}_{h}(t)\right)^{\frac{2+\delta}{2\left(\frac{N}{N-2}\right)^{h}}} \cdot\left|\mathcal{O}_{t}\right|^{1-\frac{2+\delta}{2\left(\frac{N}{N-2}\right)^{h}}}
\end{aligned}
$$

and let

$$
M_{\delta}(t):=\left(\bar{M}_{h}(t)\right)^{\frac{1}{2}\left(\frac{N-2}{N}\right)^{h}} \cdot\left|\mathcal{O}_{t}\right|^{\frac{1}{2+\delta}-\frac{1}{2}\left(\frac{N-2}{N}\right)^{h}}+1
$$

(which depends only on $\delta, t, h, N, \beta,\|f(t)\|_{L^{\infty}\left(\mathcal{O}_{t}\right)}, \lambda_{1, t}$, and $\left.\left|\mathcal{O}_{t}\right|\right)$ and $T(t, \delta, \hat{D}):=T_{h}(t, \hat{D})$ (where the constant $h$ is fixed by (4.25)), we know that, for any $t \in \mathbb{R}$, any $\mathcal{D}(\tau) \in \mathscr{D}_{\lambda_{1}}$, there exist constants $T(t, \delta, \hat{D})$ and $M_{\delta}(t)$ such that

$$
\|u(t)\|_{L^{2+\delta}\left(\mathcal{O}_{t}\right)}<M_{\delta}(t) \quad \text { for any } t-\tau \geq T(t, \delta, \hat{D})
$$




\subsection{The proof of Theorem 1.2}

Proof It is sufficient to verify the conditions of Theorem 2.4. According to Theorem 2.6, the norm-to-weak continuity is easily checked.

For any $t \in \mathbb{R}$, denote

$$
\mathcal{B}(t)=\left\{u(t) \text { satisfying }\|u(t)\|_{L^{2+\delta}\left(\mathcal{O}_{t}\right)}<M_{\delta}(t)\right\}
$$

then, according to Theorem 4.2 , for any $t \in \mathbb{R}$ and any $u_{\tau} \in D(\tau)$, there exist $\mathcal{B}(t)$ and $T(t, \delta, \hat{D})$ such that

$$
U(t, \tau) u_{\tau} \in \mathcal{B}(t)
$$

for any $t-\tau \geq T(t, \delta, \hat{D})$, that is, there exists a pullback $\mathscr{D}_{\lambda_{1}}$ absorbing set in $L^{2+\delta}(\forall \delta \in$ $[0,+\infty))$.

By Theorem 4.2, we know that, for any $\varepsilon>0$, any $\delta \in[0,+\infty)$, and each $t \in \mathbb{R}$, there exist positive constants $\bar{T}(t, \delta, \hat{D}, \varepsilon)$ and $\bar{M}$ such that

$$
\int_{\mathcal{O}_{t}\left(\left|U(t, \tau) u_{\tau}\right| \geq \bar{M}\right)}\left|U(t, \tau) u_{\tau}\right|^{2+\delta} d x<\varepsilon
$$

for any $t-\tau \geq \bar{T}(t, \delta, \hat{D}, \varepsilon)$. Combining Theorem 2.5 and Theorem 2.10, we prove that the process $U(t, \tau)$ associated to $(1.3)$ is pullback $\omega$ - $\mathscr{D}$-limit compact in $L^{2+\delta}\left(\mathcal{O}_{t}\right)$.

\section{Competing interests}

The author declares to have no competing interests.

\section{Acknowledgements}

The author would like to thank the referees for their detailed suggestions, which helped to improve the original manuscript. This work was supported by Tian Yuan Fund of Mathematics 11326100, Natural Science Foundation of Gansu Province 145RJZA033 and Gansu Province science technology plan 1606RJZA041.

Received: 28 December 2015 Accepted: 2 June 2016 Published online: 02 September 2016

\section{References}

1. Green, AE, Naghdi, PM: A derivation of equations for wave propagation in water of variable depth. J. Fluid Mech. 78, 237-246 (1976)

2. Anguiano, $M$, Caraballo, $T$, Real, $J: H^{2}$-Boundedness of the pullback attractor for a non-autonomous reaction-diffusion equation. Nonlinear Anal. 72, 876-880 (2010)

3. Łukaszewicz, G: On pullback attractors in $H_{0}^{1}$ for nonautonomous reaction-diffusion equations. Int. J. Bifurc. Chaos 20, 2637-2644 (2010)

4. Łukaszewicz, G: On pullback attractors in $L^{p}$ for nonautonomous reaction-diffusion equations. Nonlinear Anal. 73 , 350-357 (2010)

5. $L i, Y$, Wang, $S, W u, H$ : Pullback attractors for non-autonomous reaction-diffusion equations in $L^{p}$. Appl. Math. Comput. 207, 373-379 (2009)

6. Li, Y, Zhong, C: Pullback attractors for the norm-to-weak continuous process and application to the nonautonomous reaction-diffusion equations. Appl. Math. Comput. 190, 1020-1029 (2007)

7. Song, H, Wu, H: Pullback attractors of nonautonomous reaction-diffusion equations. J. Math. Anal. Appl. 325, 1200-1215 (2007)

8. Bonaccorsi, S, Guatteri, G: A variational approach to evolution problems with variable domains. J. Differ. Equ. 175, 51-70 (2001)

9. He, C, Hsiao, L: Two-dimensional Euler equations in a time dependent domain. J. Differ. Equ. 163, 265-291 (2000)

10. Kloeden, PE, Marín-Rubio, P, Real, J: Pullback attractors for a semilinear heat equation in a non-cylindrical domain. J. Differ. Equ. 244, 2062-2090 (2008)

11. Kloeden, P, Real, J, Sun, C: Pullback attractors for a semilinear heat equation on time-varying domains. J. Differ. Equ. $246,4702-4730(2009)$

12. Límaco, J, Medeiros, LA, Zuazua, E: Existence, uniqueness and controllability for parabolic equations in non-cylindrical domains. Mat. Contemp. 23, 49-70 (2002) 
13. Sun, $C$, Yuan, $Y: L^{P}$-Type pullback attractors for a semilinear heat equation on time-varying domains. Proc. R. SoC. Edinb., Sect. A 145, 1029-1052 (2015)

14. Xiao, Y, Sun, C: Higher-order asymptotic attraction of pullback attractors for a reaction-diffusion equation in non-cylindrical domains. Nonlinear Anal. 113, 309-322 (2015)

15. Zhong, C, Yang, M, Sun, C: The existence of global attractors for the norm-to-weak continuous semigroup and application to the nonlinear reaction-diffusion equations. J. Differ. Equ. 223, 367-399 (2006)

Submit your manuscript to a SpringerOpen ${ }^{\circ}$ journal and benefit from:

$\rightarrow$ Convenient online submission

Rigorous peer review

- Immediate publication on acceptance

- Open access: articles freely available online

- High visibility within the field

- Retaining the copyright to your article 